EPSC Abstracts

Vol. 14, EPSC2020-622, 2020

https://doi.org/10.5194/epsc2020-622

Europlanet Science Congress 2020

(C) Author(s) 2020. This work is distributed under

the Creative Commons Attribution 4.0 License.

\title{
Monitoring Venus cloud top: the VenSpec-U spectrometer on board ESA EnVision
}

Emmanuel Marcq ${ }^{1}$, Gabriel Guignan ${ }^{1}$, Benjamin Lustrement ${ }^{1}$, Franck Montmessin ${ }^{1}$, Jérémie Lasue ${ }^{2}$, Bruno Bézard ${ }^{3}$, Ann Carine Vandaele ${ }^{4}$, Jörn Helbert ${ }^{5}$, Thomas Widemann ${ }^{3}$, Colin Wilson ${ }^{6}$, and Richard Ghail $^{7}$

${ }^{1}$ LATMOS/IPSL, UVSQ Université Paris-Saclay, Sorbonne Université, CNRS, Guyancourt, France (emmanuel.marcq@latmos.ipsl.fr)

${ }^{2}$ IRAP, Observatoire Midi-Pyrénées, Toulouse, France

${ }^{3}$ LESIA, Observatoire de Paris, PSL, Meudon, France

${ }^{4}$ BIRA, Brussels, Belgium

${ }^{5}$ DLR, Berlin, Germany

${ }^{6}$ AOPP, Oxford, United Kingdom

${ }^{7}$ Royal Holloway, University of London, United Kingdom

\section{Instrument description}

The VenSpec-U instrument is a dual channel UV spectral imager (low and high spectral resolution channels, "LR" and "HR" hereafter), part of the VenSpec spectrometer suite on board the proposed Venus orbiter EnVision. Each channel consists of an entrance baffle, an objective composed of two lenses and a stop diaphragm, and a spectrometer composed of a slit and a toroidal holographic grating. It also includes s shortpass filter to reject the wavelengths above the higher limit of both channel bands and a zero-order trap to avoid straylight due to internal reflexions of the grating zeroorder. The optical layout is presented in Figure 1. Both LR and HR slits are parallel and the optical layout is such that both channels have the same instantaneous field of view (FoV), allowing simultaneous observations and calibrations. Each slit image is then spectrally dispersed by its respective toroidal holographic grating and is formed on a shared CMOS back-side illuminated detector.

Figure 1: VenSpec-U optical layout overview, with the $H R$ and $L R$ channels respectively on the left and on the right. 


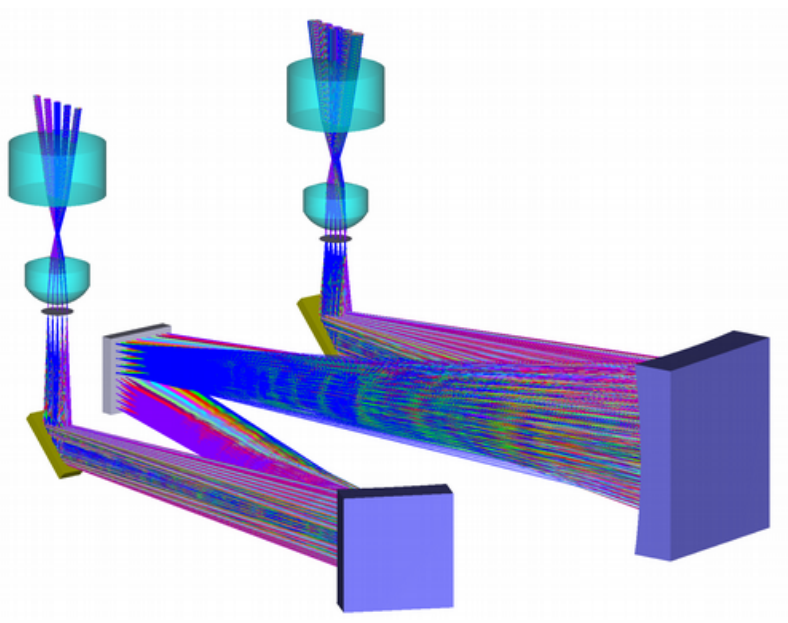

The narrow-slit axis of the detector contains the spectral information, whereas the long-slit axis contains the spatial information along the $22.5^{\circ} \mathrm{FoV}$ of each slit. The spectra of LR and HR channels are dispersed one above the other on the focal plane. The remaining spatial direction is provided through orbital scrolling ("pushbroom" strategy). Binning on the spatial axis is performed on the detector. The detector will be controlled such that the integration time and the binning scheme is adjusted independently (and simultaneously) for each channel giving high flexibility and providing parameters for the optimisation of each acquisition.

\section{Instrument Performance}

According to our forward model based on HST-STIS [Jessup et al., 2015] and SPICAV-UV heritage [Marcq et al., 2020], this shall be sufficient to measure the targeted species ( $\mathrm{SO}_{1} \mathrm{SO}_{2}, \mathrm{UV}$ absorber) with a relative accuracy better than $25 \%$. These measurements shall allow to characterize variability of Venus cloud top on temporal scales ranging from hours to years and spatial scales ranging from a few kilometers to planetary scale in relation to the climate and/or present day volcanic activity of Venus.

\section{Bibliography}

- K. L. Jessup et al., Coordinated Hubble Space Telescope and Venus Express Observations of Venus' upper cloud deck, Icarus 258, 2015

- E. Marcq et al., Climatology of $\mathrm{SO}_{2}$ and UV absorber at Venus' cloud top from SPICAV-UV nadir dataset, Icarus 335, 2020 